\title{
УДК 004.312
}

\section{МЕТОДЫ ТЕСТИРОВАНИЯ И ОТЛАДКИ ПЛИС. ИНСТРУМЕНТЫ ПРОЕКТИРОВАНИЯ ПЛИС}

\author{
Троицкий Алексей Максимович \\ магистрант \\ ФГБОУ ВО «Нижегородский государственный \\ технический университет им. Р.Е. Алексеева» \\ Научный руководитель: Окунев Александр Георгиевич \\ к.т.н., инженер \\ $\mathrm{OOO} \ll \mathrm{TEKOM»}$
}

Аннотация: Кратко рассматриваются этапы и методы разработки дизайна ПЛИС, описываются средства проектирования, их особенности. Подробнее рассматриваются методы и инструменты моделирования. Приводятся примеры тестовых модулей на языке VHDL, симуляционные диаграммы в среде проектирования Xilinx ISE. Описываются преимущества использования модели. Описываются инструменты для отладки: виртуальный логический анализатор, виртуальные входы и выходы (для Xilinx Vivado).

Ключевые слова: ПЛИС, САПР, Xilinx Ise, Vivado, этапы разработки дизайна, моделирование, отладка, VHDL, прошивка, тестбенч, несинтезируемые конструкции языка VHDL, виртуальный логический анализатор, виртуальные входы и выходы, интерфейс JTAG, MATLAB \& Simulink, $\mathrm{C} / \mathrm{C}++$.

\section{FPGA TESTING AND DEBUGGING METHODS. FPGA DESIGN TOOLS}

\section{Troitsky Aleksey Maksimovich Okunev Alexander Georgievich}

\begin{abstract}
The article discusses the stages and methods of FPGA design development, describes the design tools, their features. Modeling methods and tools are discussed in more detail. Examples of test modules in the VHDL language, simulation diagrams in the Xilinx ISE design environment are shown. The
\end{abstract}


advantages of using the model are described. Debugging tools are described: virtual logic analyzer, virtual inputs and outputs (for Xilinx Vivado).

Keywords: FPGA, Software suite, Xilinx Ise, Vivado, design development stages, s, VHDL, testbench, non-synthesizable VHDL code, synthesis, map, place and route, virtual logic analyzer, Vivado virtual inputs/outputs, JTAG interface, MATLAB \& Simulink, C/C++.

\section{Этапы разработки дизайна ПЛИС}

Рассмотрим, какие этапы проходит разработчик при разработке работоспособной прошивки FPGA. Под прошивкой будем понимать файл или файлы, необходимые для конфигурации конкретного кристалла FPGA. Фактически, прошивка - это дизайн, который прошёл стадии синтеза, определения места размещения ядер и узлов схемы, трассировки проводников внутри кристалла и определения назначения физическим выводам. Эти стадии в САПР называются соответственно - synthesis, map, place and route.

Итак, можно выделить три этапа разработки прошивки, эти этапы приведены на рисунке 1.

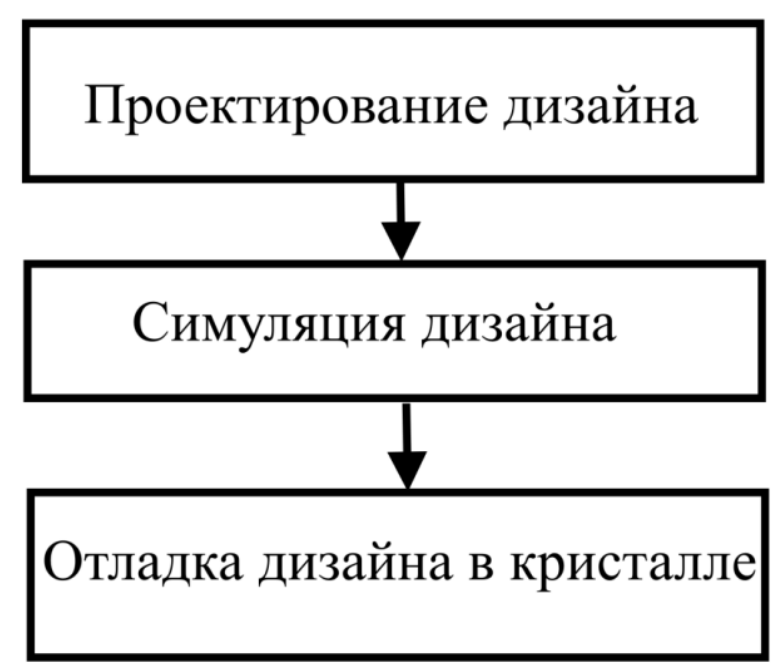

Рис. 1. Этапы разработки прошивки

Каждый из этих этапов можно разбить ещё, на стадии. Проектирование дизайна предполагает, что сначала можно смоделировать дизайн (фрагменты дизайна) используя Matlab или, например, программы на $\mathrm{C} / \mathrm{C}++$. Далее следует написание кода на языке VHDL или Verilog или, используя средства графического проектирования, также существует возможность более 
высокоуровневого проектирования, например САПР Xilinx Vivado. Также, уже на этапе проектирования следует учитывать целевое оборудование, семейство и модель кристалла. Конкретная модель FPGA может не подойти для конкретного дизайна, если не хватит ресурсов - логических ячеек, блоков имеющихся в FPGA. Отметим, что, например в Matlab и в Simulink можно использовать HDL coder, который позволяет получить из модели читаемый VHDL или Verilog код (рисунок 2). Также существуют среды разработки, поддерживающие совместное моделирование на языках HDL и C (например продукт от Aldec и Celoxica) [1, с 120], и конверторы кода C++ в HDL, Java в HDL.

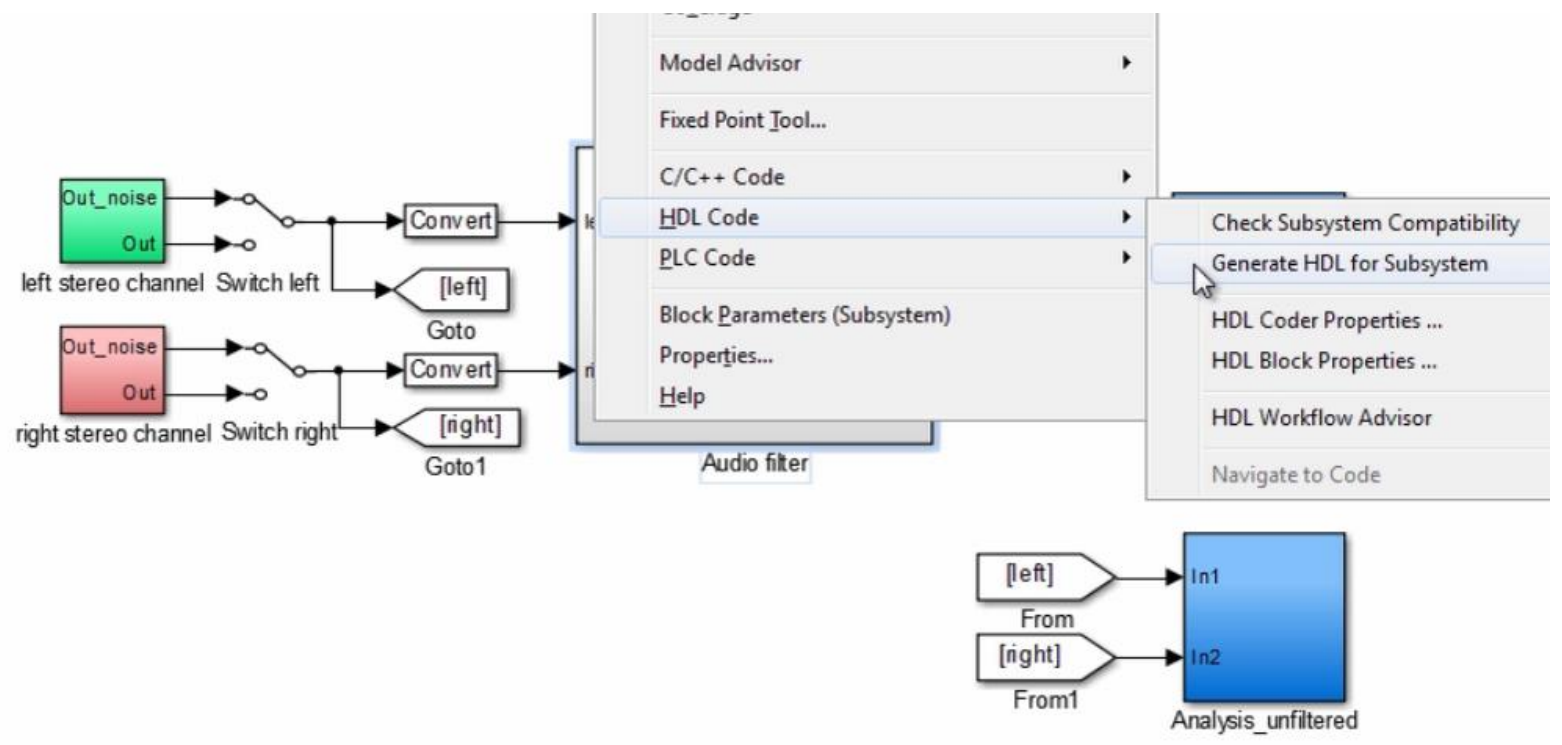

Puc. 2. HDL coder в Simulink

После проектирования дизайна следует симуляция. Формально эту стадию можно пропустить, однако на этапе симуляции намного проще увидеть правильность работы дизайна, проще найти ошибки. Также важным свойством этого этапа является возможность использовать тестбенчи, задавая любые входные воздействия, даже если они не предусмотрены в самом дизайне.

Следующий и последний этап, приведённый на рисунке 1 - отладка дизайна на кристалле. Однако, перед тем как начать отладку, необходимо подключить плату с FPGA к компьютеру, с которого будет происходить прошивка, также необходимо создать файл конфигурации физических выводов FPGA (логически соединить сигналы из дизайна с ножками кристалла, 
у которых есть номера. После этого можно использовать средства отладки, о которых будет сказано далее.

\section{Симуляция (моделирование)}

Симуляция HDL кода возможна в основных CAПР, предназначенных для проектирования FPGA: Xilinx ISE, Vivado, Altera Quartus II, Aldec ActiveHDL. При симуляции кода используются тестбенчи, логическая схема тестбенча показана на рисунке 3.

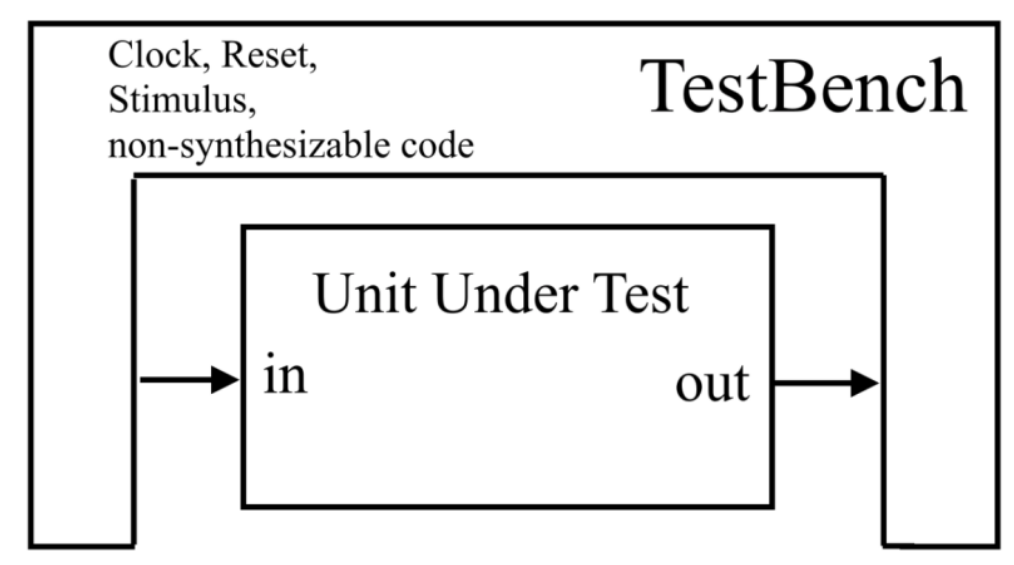

Рис. 3. Логическая структура тестбенча

Рассмотрим фрагмент дизайна в виде кода, соответствующий тестбенч и диаграммы, получаемые при симуляции в Xilinx ISE Isim. На уровне тестбенча достаточно знать только описание объекта моделирования, находящегося на верхнем уровне иерархии [2, с 111]. В нашем примере на выходе верхнего модуля будут сигналы led_1 и led_2, как если бы мы хотели помигать двумя светодиодами (рисунок 4).

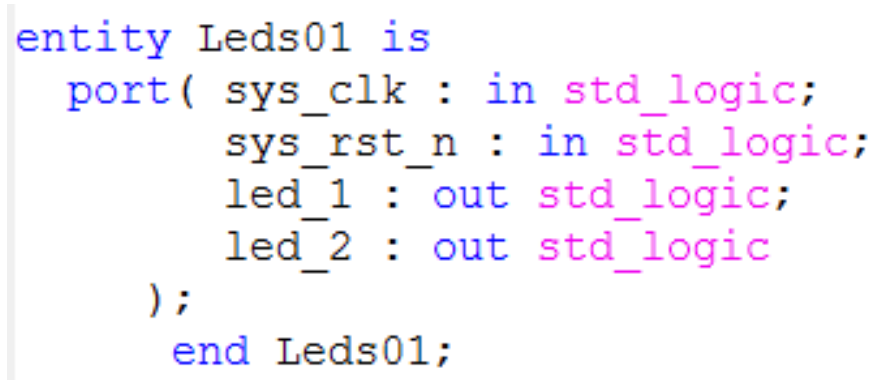

Рис. 4. Декларация объекта моделирования

Теперь сгенерируем тестбенч в автоматическом режиме, выбрав тип добавляемого в проект файла - VHDL Test bench. Архитектура тестбенча будет иметь вид, приведённый на рисунке 5. 


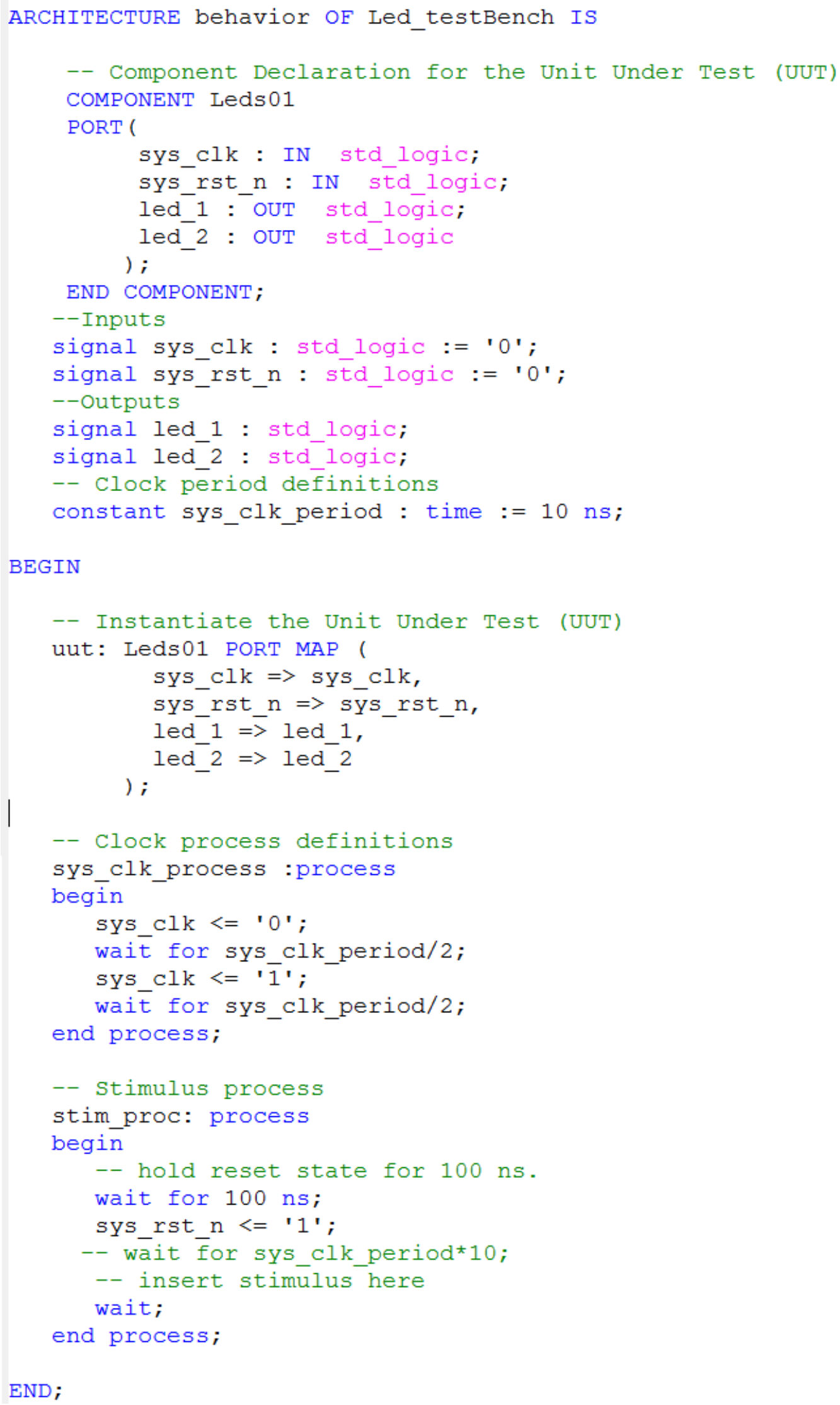

Рис. 5. Описание архитектуры тестбенча 
Как видно из рисунка 5, тестбенч будет включать следующие элементы управляющие сигналы для задания входных воздействий (сигналы sys_clk, sys_rst_n) и константу, относительно которой в процессе sys_clk_process определяется поведение sys_clk). В тестбенче можно задать любое требуемое входное воздействие. Диаграммы для нашего примера будут иметь вид, приведённый на рисунке 6.

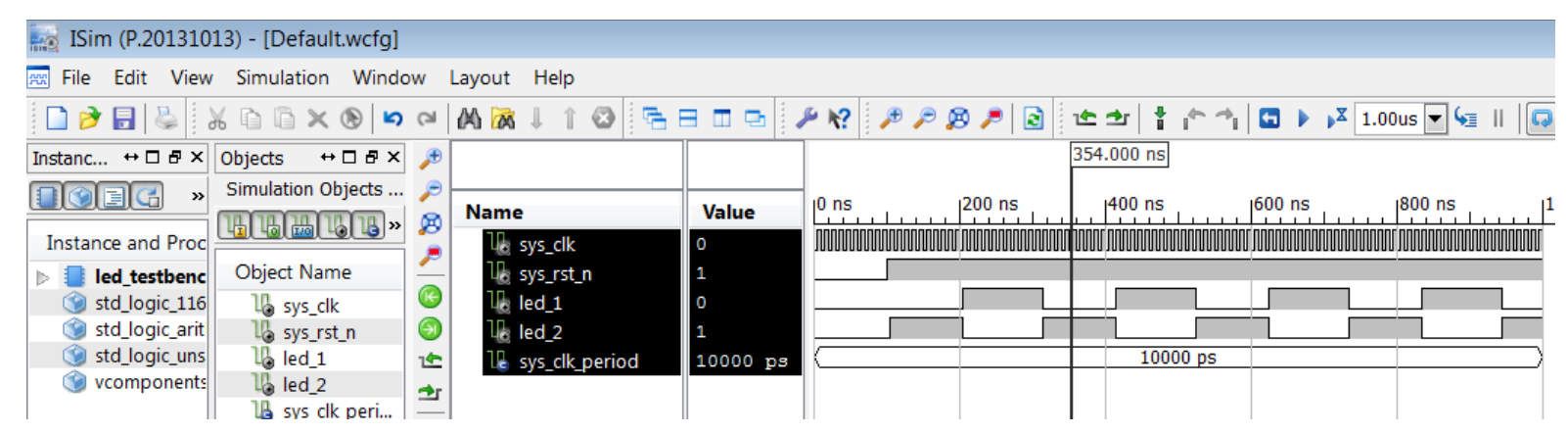

Рис. 6. Симуляция дизайна с использованием тестбенча

Как указано на рисунке 3 , тестбенч может включать несинтезируемые конструкции. Одна из таких - конструкция с использованием ключевых слов VHDL: file, acsess [3, с 69]. Используя эти ключевые слова можно организовать запись и чтение из файла. Использование несинтезируемых констукций упрощает разработку на этапе симуляции.

Используя Xilinx ISE ISim, можно определять управляющие сигналы не изменяя код с помощью функций Force Clock, Force Constant. Результат переопределения управляющего сигнала sys_clk показан на рисунке 7 . Выбраны параметры: isim force add \{/led_testbench/sys_clk\} 1 -radix bin -value 0 radix bin -time $20 \mathrm{~ns}$-repeat $40 \mathrm{~ns}$-cancel $500 \mathrm{~ns}$.

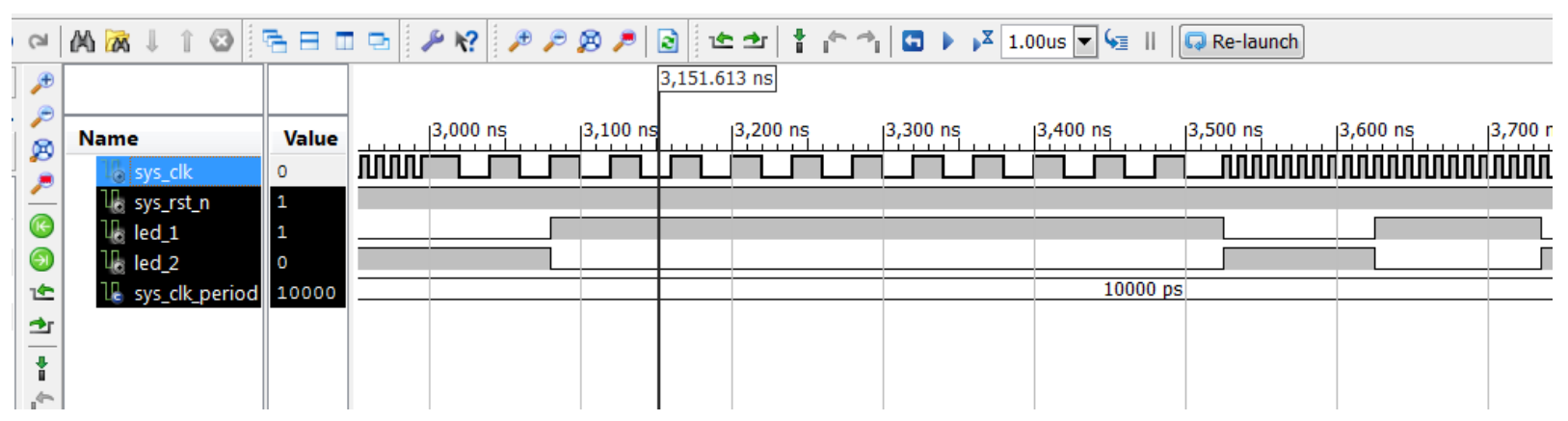

Рис. 7. Переопределение управляющих сигналов с помощью force clock 


\section{Отладка прошивки}

После успешной проверки дизайна на работоспособность при симуляции, можно сгенерировать прошивку (с помощью САПР) и сконфигурировать конкретную ПЛИС в конкретной плате. Однако безошибочное поведение дизайна в симуляции не гарантирует того, что в реальном устройстве всё будет работать правильно. Причины могут быть различные. Внешние по отношению к кристаллу ПЛИС: неправильное подключение внешних устройств, неправильная конфигурация внешних устройств. Ошибки в дизайне: например, ошибки связанные с инерционными, транспортными задержками, дельтазадержками, с разрешением неоднозначности установления сигнала (при перегруженности чипа) [2, с 89].

Одна из проблем, связанных с отладкой заключается в отсутствии возможности увидеть воочию, что происходит внутри ПЛИС [4, с 225]. Если использовать наиболее очевидный способ отладки - посмотреть напряжения на выводах, то возникнут две проблемы:

1. Высокая частота, с которой изменяются значения сигналов (невозможно рассмотреть происходящее на экране осциллографа).

2. Может не хватить свободных ножек, чтобы вывести все исследуемые сигналы.

По этим причинам, чаще применяется другой способ отладки использование виртуального логического анализатора. Идея заключается в использовании некоторого количества ресурсов ПЛИС для реализации блока логического анализатора, который фиксирует активность заданных сигналов. Эти данные могут быть сохранены в блоках встроенного ОЗУ ПЛИС, доступ к содержимому которых может быть получен с помощью внешнего программного обеспечения через JTAG-порт [4, с 228] (рисунок 8). 


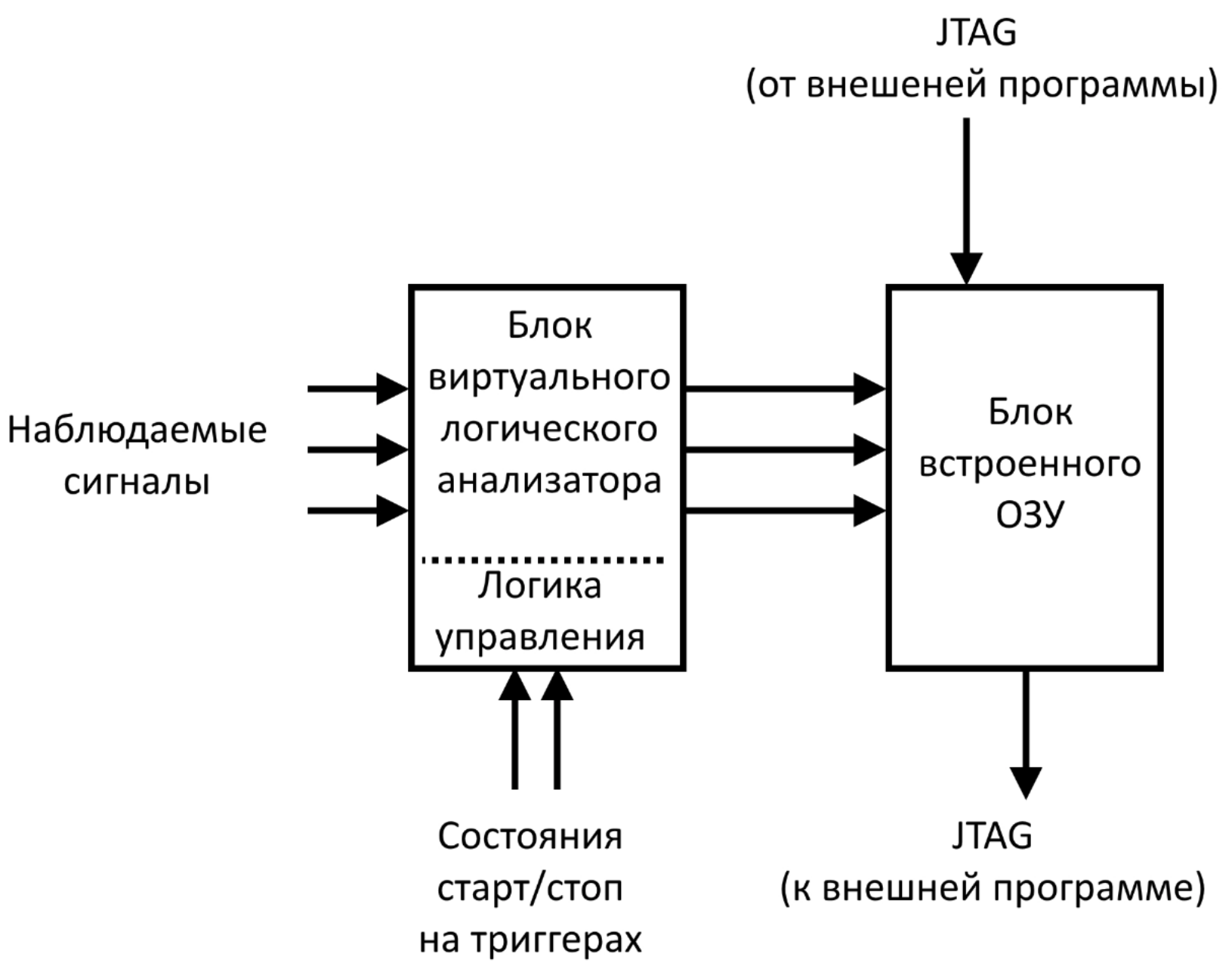

Рис. 8. Использование виртуального логического анализатора

Программное обеспечение для работы с логическим анализатором часто поставляется вместе со средствами проектирования. Для Xilinx ISE это Chipscope Pro, для Quartus II - SignalTap. B Xilinx Vivado инструмент работы с логическим анализатором встроен. B Vivado существует ещё один инструмент отладки - виртуальные входы/выходы (рисунок 9). И помощью виртуальных входов/выходов можно во время отладки, не осуществляя перепрошивку, изменять значения на выходных сигналах, которые можно подключить к исследуемому компоненту в дизайне [5, с. 25]. 


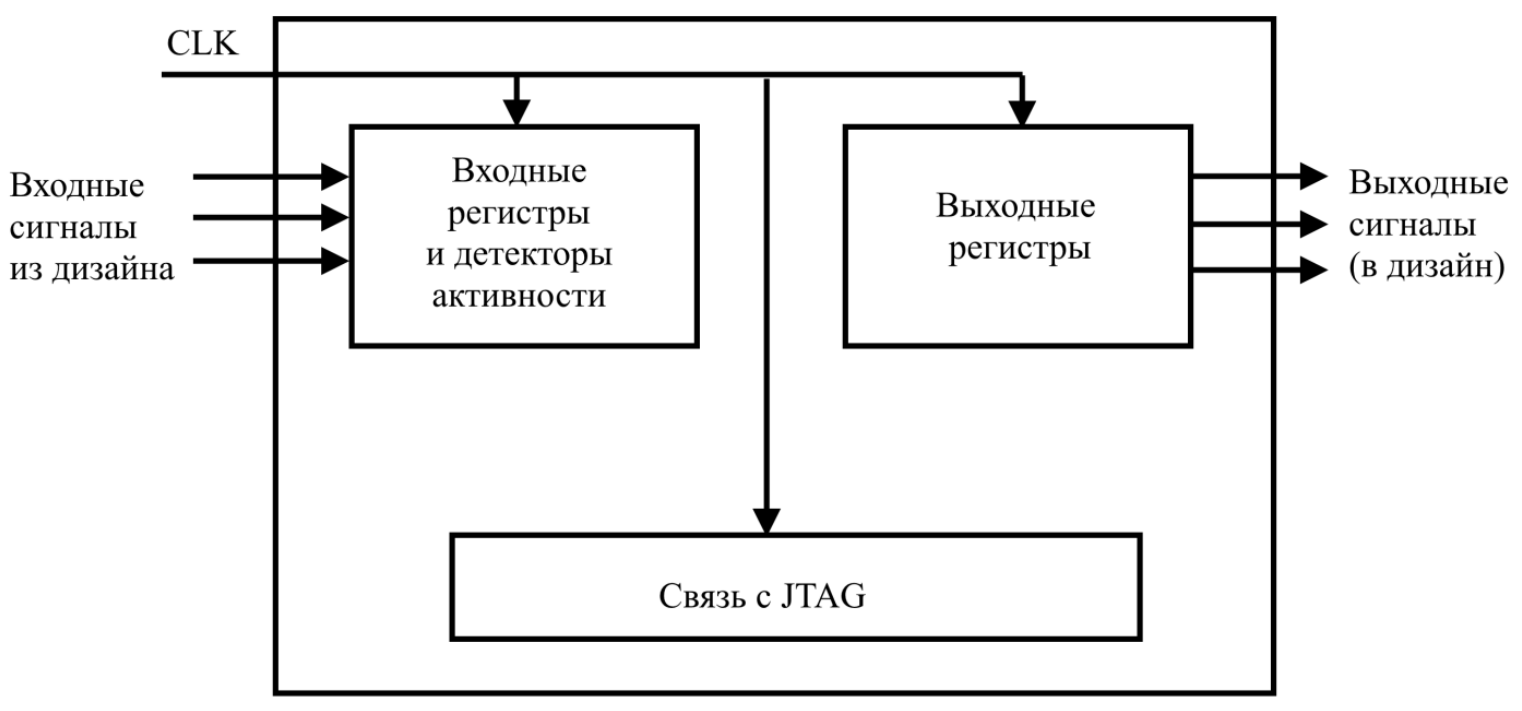

Puc. 9. Virtual Input/Output (VIO) core

\section{Список литературы}

1. Компоненты и технологии, №1'2004. Михаил Долинский. Обзор современных подходов и средств к «программистской» разработке

2. Проектирование цифровых систем на VHDL. Е.А. Суворова, Ю.Е. Шейнин. Издательство: БХВ-Петербург 2003.

3. Современная электроника №5 2008. СТА-Пресс. Пётр Бибило. О несинтезируемых конструкциях языка VHDL.

4. Проектирование на ПЛИС: Архитектура, средства и методы. Курс молодого бойца. Максфилд Клайв. (Москва: Издательский дом «Додэка-XXI», 2007. - Программируемые системы).

5. UG936 (v2019.2) December 10, 2019. Vivado Design Suite Tutorial. Programming and Debugging.

6. Цифровая схемотехника и архитектура компьютера. Второе издание. Дэвид М. Хэррис и Сара Л. Хэррис. Morgan Kaufman. English Edition 2013.

7. UG904 (v2021.1). August 30, 2021. Vivado Design Suite User Guide. Implementation.

8. Проектирование встраиваемых микропроцессорных систем на основе ПЛИС фирмы Xilinx. Зотов. Ю.В. 2017 г. 520 стр.

9. M. Haldar A. Nayak N. Shenoy A. Choudhary and P. Banerjee FPGA Hardware Synthesis' from MATLAB September 2000.

10. Поляков А.К. Языки VHDL и VERILOG в проектировании цифровой аппаратуры. М.: СОЛОН Пресс, 2003. 